\title{
The Monitoring System of Water Environment Based on Overlay Network Technology ${ }^{*}$
}

\author{
Xueliang Fu, Changyou Li, Buyu Wang, Honghui Li, Hailei Ma, \\ and Dongnan Zhu \\ Inner Mongolia Agricultural University, Hohhot, 010018, P.R. China \\ Fuxleimau.edu.cn
}

\begin{abstract}
At present, although methods of automatic, periodical manual data collection have been adopted in some areas, there are many problems existing in these methods. This paper proposes a new framework for online monitoring of water environment based on overlay network technology. Especially, we build the multi-level overlay network, which consists of the GPRS networks, mobile networks and internet networks. Following this, a multi-dimensional data cube for water environment is established using the ETL process with the input of complex heterogeneous data collected. Thereby, the framework of data center is established for on-line early warning of water environment and data analysis and processing. This framework has been put into use in Wuliangsuhai for the on-line real-time water monitoring tests. The results show that the overlapping network architecture is effective and on-line analysis and early warning of heterogeneous data is efficient.
\end{abstract}

Keywords: WSN, Overlay network, ETL, Multidimensional data cube.

\section{Introduction}

Currently, water-quality monitoring in Wuliangsuhai is mainly performed manually [1]. Researchers in related institutes reside in Wuliangsuhai, and gather water-quality data manually during the ice-absent period. The main method to gather data relies on portable instruments. It is difficult to meet the needs of comprehensive monitoring, analysis and early warning in Wuliangsuhai for water-quality. Therefore, the comprehensive development of research and eco-social benefit is seriously restricted [2].

There are mainly four issues in the above traditional method. Firstly, researchers had to be outside for long time, thus, they cannot conduct large-scale experimental analysis using tools and computing environment. Secondly, the continuity of data collection is poor, and a sample space is very limited. In the ice-absent period, one can only collect data once or twice per day (once at noon, or twice in the morning and evening respectively). One cannot collect data at night as well as in freeze-up period.

\footnotetext{
* The research is supported by Chinese Natural Science Foundations $(50969005,40901262)$ and by Specialized Research fund of High Education for Inner Mongolia (Njzy08046).
} 
So, the number of the achieved water-quality data is no more than 200 records. Therefore, it is impossible of widely data mining and analysis owing to the significant shortage of data. Moreover, the quality of the collected data cannot be guaranteed. Thirdly, using the manual-collecting method, the quality of data is effected strongly by the professional proficiency of the researchers and the usage of the equipment. As a result, dirty data may appear and cannot be analyzed and rectified using other timesequential data. Finally, the collection area is very limited. Due to the broad water area of the Wuliangsuhai Lake, it is impossible for the residing researchers to collect data at the same time from multiple locations far away from each other. As a result, there is no way to analyze and compare data in terms of time scale and location.

The current trends to resolve these issues is to use the framework of cloud services, with which data is online automatically assembled, real-time transmitted using overlay networks. Decision of knowledge is made in a data center.

Wireless sensor networks (WSN) are the task-oriented wireless networks which consist of a number of wireless sensor nodes [3]. WSN integrates multiple area technology, including sensor technology, embedded computing technology, modern networking, wireless communication technology, distributed information processing technology and others. In WSN, various micro sensors take responsibility of on-line monitoring target; the embedded computing resources take care of processing data obtained by sensors; the related information is send to the remote user data center using wireless communication networks. This technology can be broadly applied in the military defense, industrial and agricultural controls, urban management, biological medicine, environmental monitoring, disaster relief, antiterrorism and remote control in dangerous areas. It is attractive in both academy and industry [4].

This paper studied the design of monitoring system for environment of water based on the overlapping networks in order to deal with the issues about Wuliangsuhai water-quality monitoring.

\section{Main Contributions}

\subsection{Architecture}

Our proposed system is mainly composed of overlapping network communication subsystem, the water-quality sensor acquisition subsystem, multi-dimension data analysis subsystem [5] and solar power subsystem. The issues are addressed using multi-layer overlay network technologies on inter communication among networks, such as 485 network, GPRS network, PSTN network and computer networks. In this system, the task-oriented data-collecting network is constructed using water quality sensors and data acquisition equipment based on wireless sensor network technology; the multi-dimensional data analysis center are established based on OWB and ETL technologies for early warning and comprehensive analysis of instantaneous data and historical data; solar power system is employed for 24-hour uninterrupted power supply all day long and thus provide guaranteed environments for the good performance of the entire system. System architecture is shown in Figure 1. 


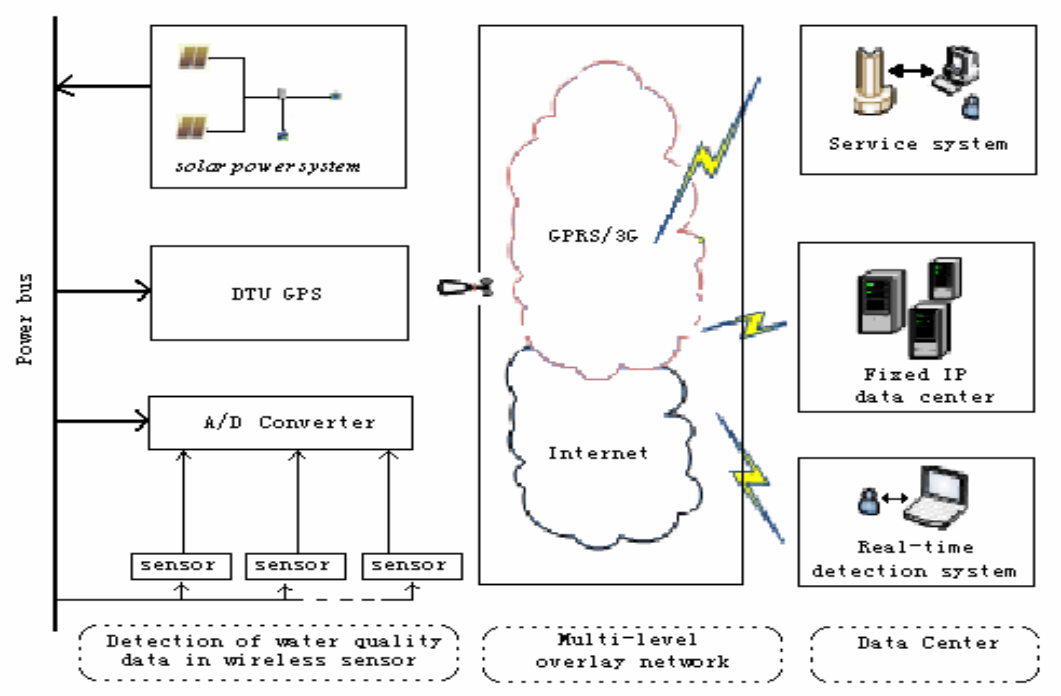

Fig. 1. The system architecture

\subsection{Overlay Network Design}

A networking framework which enables multi-network integration must be explored in order to realize a distributed resource management platform. This platform can provide for the application layer reliable and efficient resource searching and localization service through monitoring link status, shielding network failures and changes. Also, this platform can provide for application service such as network routing optimization through node detection of network paths and performance.

The real-time signal data on water quality parameters is achieved through the data acquisition subsystem. The collected signal data is first send to a GPRS-wireless hybrid communication system via a RS485 network, and then transfer to the data center with fixed IP address by the GPRS wireless network. In the data center, the signal data is translated, analyzed, processed and stored. The TCP multicast protocol, i.e., "center to multi-point", is adopted for reliable and transparent data transmission.

Multiple-layer hybrid overlay networks are constructed between each sensor node, which registers as an online communication node and the super node in the data center with a fixed IP. The super node manages the whole WSN. Each facility, which is eligible for the WSN, can log in, exit and abnormal exit through this subsystem. This subsystem is important for the establishment of a reliable network, which provides bidirectional data transmission channels and communication links for the transmission of signal data and control signaling.

The layered hybrid network architecture is exploited in the whole network framework. The whole network is divided into four layers: the service layer, the core layer, the access layer and the link layer. 


\subsection{Data Stream Processing}

The Data center architecture is shown in Figure 2. The data stream processing includes two steps: receiving data stream and sending data stream, which are described as follows:

1) Receiving data stream

i. The on-line real-time data on water quality parameters is send to the data center in the sampling frequency using the data acquisition subsystem. The acquisition subsystem consists of water-quality-parameter sensors, filters and A / D converters.

ii. After receiving the real-time data, the data center analyzes the data logically, eliminate the dirty data, and store the qualified data in the transient database.

iii. Water-quality analysis-type database or data warehouse, which is suitable for analysis and statistics, is built from the data in transient database using the ETL tools combined with the functionalities of operations / scheduling of enterprise database.

iv. Various analysis products needed by users can be generated using the database products for analysis (BI data warehouse reporting engine) together with the waterquality analysis-type database or data warehouse.

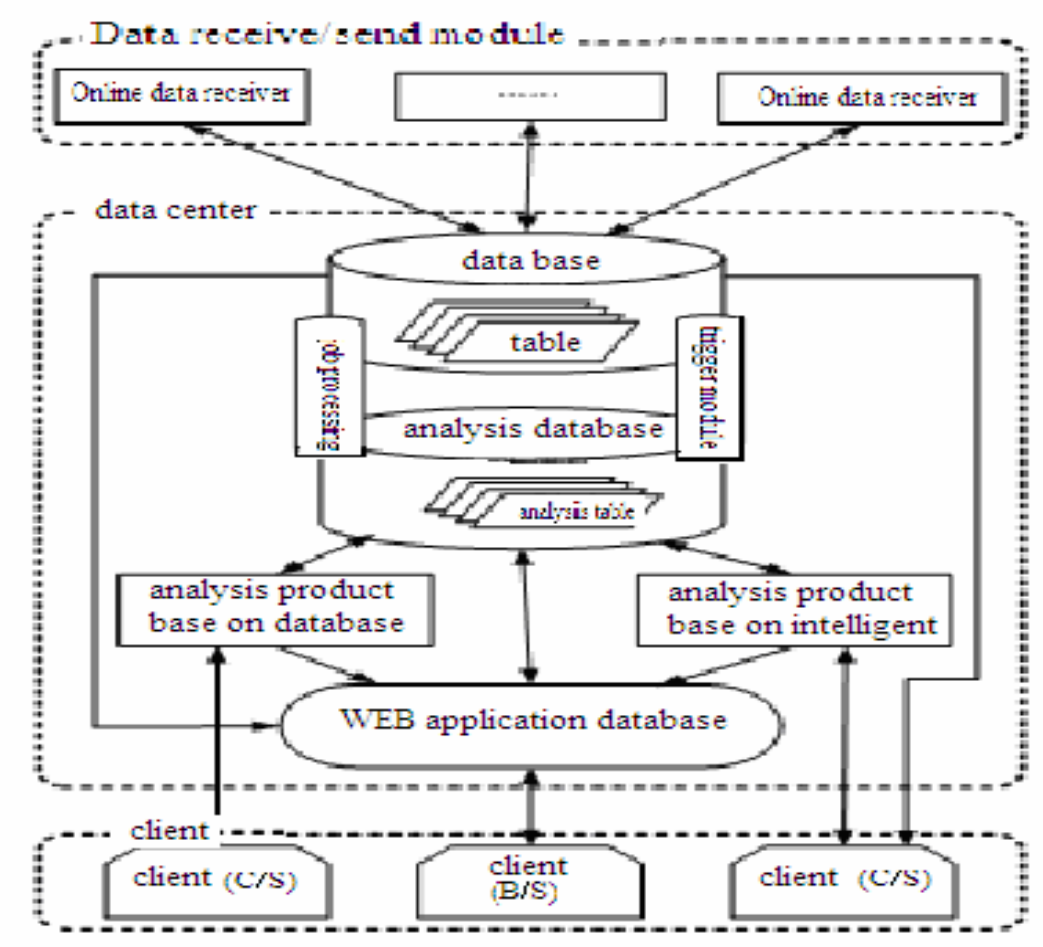

Fig. 2. Data center architecture

2) Sending data stream

i. A user can log in the data center using the mobile terminal equipment (e.g., mobile phones, PDA, etc.) or the water quality data viewer on a fixed terminal. Through 
the data center, the user can transmit the acquisition subsystem in order to set the sampling period and other parameters of the acquisition system.

ii. With the help of the data center for sending the control information, an eligible user can turn on or off the power supply system, or remote manage the power supply system.

\section{Experiment Setup and Discussion}

Firstly this paper deployed the PH-value and ORP and oxygen-content sensors in the Wuliangsuhai Lake. Then, the real-time, automatic data acquired with adjustable sampling frequency has been achieved using the proposed water-quality sensor acquisition subsystem, the overlay network transmission subsystem, and the data storage and processing subsystem. Thereby, the water-quality parameter data can be processed and analyzed in a very short time.

The software in PLC (Programmable Logic Controller) exploits the means of center-to-multi-point communication in the water-quality-parameter signal acquisition subsystem in Wuliangsuhai; the Baud Rate is 9600; the survivable mechanism is achieved through the heart-rate packet; the communications signaling employs ASCII-code signaling. The connections between the PC software and the data center are established using TCP/IP protocol. The unity of the user programming interface is approached through encapsulating the Socket package in the system level and the integrated converged communications from the data link layer, the network layer and the application layer.

The data center is built based on Oracle database, where the transaction concurrency mechanism and trigger mechanism are used, and the data OLTP is achieved through the job scheduling. Oracle DWB is used to build the data warehouse which provides analysis environments. All analysis products and user UI are implemented using the B/S framework; the system is built using the SSH enterprise multi-layer computing framework.

After the deployment in practice and the simulations, it is found that the proposed overlay network framework is sensitive very much to the strength of the data signals. The instantaneous packet loss may suffer when the signals become weak, which results in dirty data. However, due to the relatively high sampling frequency, this can be ignored in the context of the research and the application on water environment monitoring only if the data is accurate in the time granularity of an hour.

\section{Conclusion}

This paper proposed and implemented the Monitoring system of water environment based on overlay network technology in order to deal with the issues on the current water environment monitoring in the Wuliangsuhai Lake. Through the field deployment of our proposed system, simulation results verify that the effectiveness of our design. It solves a series of issues existing in the current monitoring method mentioned before. The future work is to investigate the issues related to data processing and data reliable transmission both in academy and in practice. 


\section{References}

[1] Seelig, H.D., Hoehn, A., Stodieck, L.S., Klaus, D.M., Adams III, W.W., Emery, W.J.: Relations of remote sensing leaf water indices to leaf water thickness in cowpea, bean, and sugarbeet plants. Remote Sensing of Environment 11(2), 445-455 (2008)

[2] Ross, B., Steiner, G., Kiesshauer, Bradter, M., Cammann, K.: Instrument with integrated sensors for a rapid determination of inorganicions. Sensors and Actuators 27, 380-383 (2009)

[3] Tatyana, B., Thomas, A.C., Thomas, H.C.: A sensitive nitrate ion-selective electrode from a pencil lead. Journal of Chemical Education 82(3), 439-441 (2009)

[4] Wang, B., Li, M.: A clustering Algorihm Based on Latent Semantic Model. In: IEEE ICACIAP 2009, October 2009, pp. 44-48 (2009)

[5] Cooley, P.M., Barber, D.G.: Remote Sensing of the Coastal Zone of Tropical Lakes Using Synthetic Aperture Radar and Optical Data. Journal of Great Lakes Research 29(2), 62-75 (2003)

[6] Wang, Y., Dong, W., Zhang, P., Yan, F.: Progress in Water Depth Mapping from Visible Remote sensing Data. Marine Science Bulletin 26(5), 92-101 (2007) 